Research Article

\title{
Serum Interleukin-23 in Polish Patients with Systemic Lupus Erythematosus: Association with Lupus Nephritis, Obesity, and Peripheral Vascular Disease
}

\author{
Katarzyna Fischer, ${ }^{1}$ Hanna Przepiera-Będzak, ${ }^{2}$ Marcin Sawicki, ${ }^{3}$ Anna Walecka, ${ }^{3}$ \\ Iwona Brzosko, ${ }^{1}$ and Marek Brzosko ${ }^{2}$ \\ ${ }^{1}$ Independent Laboratory for Rheumatologic Diagnostics, Pomeranian Medical University in Szczecin, Unii Lubelskiej 1, \\ 71-252 Szczecin, Poland \\ ${ }^{2}$ Department of Rheumatology, Internal Medicine and Geriatrics, Pomeranian Medical University in Szczecin, Unii Lubelskiej 1, \\ 71-252 Szczecin, Poland \\ ${ }^{3}$ Department of Imaging Diagnostics and Interventional Radiology, Pomeranian Medical University in Szczecin, Unii Lubelskiej 1, \\ 71-252 Szczecin, Poland
}

Correspondence should be addressed to Katarzyna Fischer; katarzyna.fischer11@gmail.com

Received 23 June 2017; Revised 7 November 2017; Accepted 27 November 2017; Published 21 December 2017

Academic Editor: Rosa E. Navarro-Hernandez

Copyright ( 92017 Katarzyna Fischer et al. This is an open access article distributed under the Creative Commons Attribution License, which permits unrestricted use, distribution, and reproduction in any medium, provided the original work is properly cited.

\begin{abstract}
Objectives. To analyze the correlation between the serum concentration of interleukin- (IL-) 23 and atherosclerotic changes, traditional atherosclerotic risk factors, the autoantibody profile, and involvement of selected organs in systemic lupus erythematosus (SLE) patients. Patients and Methods. We studied 94 SLE patients and 27 controls. We analyzed the IL-23 serum concentration, autoantibodies, carotid intima-media thickness and atherosclerotic plaque, the ankle-brachial index, atherosclerotic risk factors, and organ manifestations. Results. Concentrations of IL-23 significantly differed between SLE patients and the controls $(p=0.0015)$. On the basis of multivariate stepwise analysis, we revealed that high levels of IL-23 were associated with atherosclerotic plaque in common femoral arteries (OR $=12.67 ; 95 \% \mathrm{CI}$ : 1.41-113.84), lupus nephritis $(\mathrm{OR}=3.69 ; 95 \% \mathrm{CI}: 1.16-12.22)$, and obesity $(\mathrm{OR}=4.21 ; 95 \% \mathrm{CI}: 1.40-12.67)$. Autoantibodies related to IL-23 were antiphosphatidylethanolamine antibodies $(\mathrm{OR}=11.06$; 95\% CI: 1.24-98.65) and anti-SS-B/La antibodies $(\mathrm{OR}=15.43 ; 95 \% \mathrm{CI}$ : 1.73-137.25). Conclusions. IL-23 may be involved in lupus nephritis pathogenesis. Through its association with obesity and selected antiphospholipid antibodies, IL-23 might promote a hypercoagulable state contributing to atherothrombosis development in SLE patients.
\end{abstract}

\section{Introduction}

Systemic lupus erythematosus (SLE) is a chronic, inflammatory, multisystem autoimmune disease characterized by an irreversible break in immunologic self-tolerance and successive immune-mediated tissue damage [1]. A remarkable feature of the disease is the clinical heterogeneity, which indicates variations in underlying etiologic factors. The multifactorial etiology of SLE includes genetic susceptibility and hormonal and environmental triggers [2, 3]. Defective function of $\mathrm{T}$ cells and overactivation of $\mathrm{B}$ cells as well as defective clearance of apoptotic debris cause the production of autoantibodies, activation of complement, formation and deposition of immune complexes, and, consequently, tissue and organ damage $[4,5]$. The crucial role in SLE pathogenesis plays innate and adaptive immune dysregulation, and it has been confirmed that certain cytokines are closely linked to SLE pathogenesis [6].

Interleukin- (IL-) 23 belongs to the IL- 12 family cytokines and consists of two subunits, p19 and p40 [7]. IL-23 is produced by macrophages, dendritic cells, keratinocytes, and other antigen-presenting cells and through its 
interaction with the IL-23 receptor plays a central role in inflammation including the induction of Th17 cells $[8,9]$. The IL-23-IL-17 axis is emerging as a critical regulatory system that bridges the innate and adaptive arms of the immune system and plays a critical role in development of autoimmune inflammatory diseases [10]. In fact, there are reports showing increased serum levels of IL-23 in systemic sclerosis [11], rheumatoid arthritis [12], primary antiphospholipid syndrome (PAPS) [13], spondyloarthropathies [14, 15], and inflammatory bowel diseases [16]. IL-23 has also been implicated in SLE [17-20], atherosclerosis [21, 22], and obesity [23].

However, to our knowledge, no complex data are available considering the additional relationship between serum concentrations of IL-23, atherosclerosis, and atherosclerotic risk factors in SLE patients.

\section{Objectives}

The aim of this study was to evaluate levels of IL-23 and their association with atherosclerotic changes, traditional atherosclerosis risk factors, disease characteristics including autoantibody profiles, and the involvement of selected organs in SLE patients.

\section{Materials and Methods}

This study was approved by the Local Ethics Committee of Pomeranian Medical University in Szczecin. Informed consent was obtained from all patients.

All patients were Caucasian. We studied 94 SLE patients and 27 healthy volunteers as the controls. The diagnosis of SLE was made according to the 1997 American College of Rheumatology Revised Criteria for Classification of Systemic Lupus Erythematosus [24].

The following data were recorded: age, gender, disease duration, activity of the disease according to Systemic Lupus Erythematosus Activity Index (SLEDAI) [1], antiphospholipid syndrome (APS) [25], lupus nephritis, cerebrovascular manifestations (transient ischemic attacks (TIA), stroke) and cardiovascular manifestations (coronary artery disease (CAD), myocardial infarction (MI)), Raynaud's phenomenon, and vasculitis.

All SLE patients and matched controls underwent noninvasive imaging investigations in the Department of Diagnostic Imaging and Interventional Radiology Pomeranian Medical University in Szczecin. All analyses were performed by the same experienced ultrasonographist with HDI 3500 (ATL) using a $5-12 \mathrm{MHz}$ linear transducer. Carotid intimamedia thickness (cIMT) measurements were performed with B-mode ultrasound in common carotid artery, bifurcation, and internal carotid artery on the right and left sides according to procedures previously described [26]. Due to the high variability of this parameter in populations $[27,28]$, the normal and pathological ranges of cIMT values were established on the basis of measurements in the controls [29]. The B-mode ultrasound was also used as a screening for atherosclerotic plaque presence in carotid and lower extremity arteries (the iliac, common femoral, deep femoral, superficial femoral, popliteal, and tibial arteries) [30]. The ankle-brachial index was assessed using Doppler ultrasonography and calculated as a ratio of systolic pressure measured in the posterior tibial and dorsal arteries of both feet to the systolic pressure in the brachial artery. Abnormal values were considered at $\mathrm{ABI}<1.0$ [31].

We assessed the presence of traditional risk factors of atherosclerosis: hypertension (systolic blood pressure $\geq$ $140 \mathrm{mmHg}$, diastolic pressure $\geq 90 \mathrm{mmHg}$ ), dyslipidemia (total cholesterol $>190.0 \mathrm{mg} / \mathrm{dL}$, LDL cholesterol $>115.0$ $\mathrm{mg} / \mathrm{dL}$, HDL cholesterol in males $<40.0 \mathrm{mg} / \mathrm{dL}$ and in females $<45.0 \mathrm{mg} / \mathrm{dL}$, and triglycerides $>150.0 \mathrm{mg} / \mathrm{dL}$ ), overweight and obesity based on body mass index (BMI) (overweight when BMI was 25 to $<30$, obesity when BMI was $\geq 30$ ), diabetes, smoking habits, oral contraceptive use, and positive family history of cardiovascular diseases.

Blood was taken for the assessment of the erythrocyte sedimentation rate (ESR) (Westergren method) and Creactive protein (CRP) (turbidimetric nephelometry), uric acid (modified Trinder assay based on the methods of Trivedi and Kabasakalian), homocysteine (fluorescent polarization immunoassay), total cholesterol (enzymatic, based on the formulation of Allain et al. and the modification of Roeschlau), direct LDL (low-density lipoproteins), direct HDL (high-density lipoprotein) cholesterol (enzymatic, colorimetric), direct triglycerides (enzymatic, colorimetric), glucose (hexokinase-mediated reaction), and fibrinogen (Clauss method).

The serologic diagnostics included the profile of autoantibodies determined with indirect immunofluorescence assay (IIFA), sandwich enzyme-linked immunosorbent assay (ELISA), and coagulation tests. IgG antinuclear antibodies (ANA) were assessed on the HEp-2 cell line contaminated by CVCL_0030 cervical adenocarcinoma human HeLa using the IIFA technique and monospecific tests performed with the ELISA method for detection of anti-double stranded DNA, anti-nucleosome, anti-Sm, anti-SS-A/Ro, anti-SS-B/La, anti-ribosomal $\mathrm{P}$ protein, anti-histone, and anti-U1-RNP antibodies (EUROIMMUN AG Medizinische Labordiagnostika tests, Lubeck, Germany). Antiphospholipid antibodies (aPLs) were determined with the ELISA method. The aPLs profile consisted of anticardiolipin (aCL) IgG, IgM, and IgA; anti-beta2-glycoprotein I IgG, IgM, and IgA (EUROIMMUN); antioxidized low-density lipoprotein IgG and IgM (IMTEC Immunodiagnostika, Berlin, Germany); antiprothrombin IgG, IgM, and IgA (AESKU.DIAGNOSTICS, Wendelsheim, Germany); antiphosphatidylserine (aPS) IgG and IgM (Demeditec Diagnostics, Kiel, Germany); antiphosphatidylethanolamine (aPE) IgG and IgM (The Binding Site, Birmingham, UK); and lupus anticoagulant (LA) performed with coagulation methods according to the International Society of Thrombosis and Haemostasis criteria [25]. The profile of anti-neutrophil cytoplasmic antibodies (ANCA) included screening IIFA for cytoplasmic (C-ANCA) and perinuclear (P-ANCA) and monospecific tests performed with the ELISA method for detection of anti-proteinase 3, anti-myeloperoxidase, anti-lactoferrin, anti-elastase, and anti-BPI antibodies (EUROIMMUN). The anti-endothelial cell antibodies were tested with 
human umbilical vein endothelial cells using the IIFA method (EUROIMMUN).

Serum was stored at $-80^{\circ} \mathrm{C}$ until analysis for IL-23 using the sensitive ELISA method with the Quantikine Human IL23 Immunoassay ELISA kit (minimum detectable dose less than $6.8 \mathrm{pg} / \mathrm{mL}$ ). Kits were from R\&D Systems, Minneapolis, USA. The system uses microplates coated with a monoclonal antibody and an enzyme-linked polyclonal antibody specific for IL-23. All analyses and calibrations were performed in duplicate and were read using BioTek PowerWave XS, BioTek Instruments, Winooski, USA.

All continuous variables were checked for equality distribution with Kolmogorov-Smirnov test. Data are described as the mean \pm standard deviation and median (Q1, Q3). Comparison of continuous variables was performed by the Mann-Whitney $U$ test. For categorical variables, differences were assessed by the logistic regression model and multivariate stepwise analysis. The logistic regression model and multivariate stepwise analysis probability $(P)$ were assessed by a chi-square testing or Fisher's exact test. Results were shown as a $P$ value, odds ratio (OR), and $95 \%$ confidence interval (95\% CI) adjusted for sex and age. Findings were considered statistically significant at $P<0.05$. Additionally, Bonferroni correction was used for related variables. All statistical analyses were performed with STATISTICA version 8.0, StatSoft Inc., Tulsa, OK, USA.

\section{Results}

The clinical and laboratory characteristics of the patients and healthy controls, including classical atherosclerotic risk factors and serologic profile, are presented in Tables 1, 2, 3, 4, and 5. The study group consisted of 82 women and 12 men suffering from SLE. The mean age was 44.5 years. The disease duration ranged from 1 to 30 years. The majority of patients presented low and medium SLE activity indices (52 and 33, resp.). The coexistence of APS was confirmed in 31, kidney involvement in 24, stroke in 10, and CAD in 11 SLE patients. The analysis of classical atherosclerosis risk factors revealed abnormal levels of total cholesterol in 52, LDL cholesterol in 40, HDL cholesterol in 5, and triglycerides in 25 patients with SLE. Hypertension was present in 37, overweight in 15 , obesity in 23 , diabetes in 11 , smoking habits in 31 , and positive family history of cardiovascular diseases in 4 SLE patients. The use of oral contraceptives confirmed 4 women suffering from SLE. Table 5 shows the serological profile of SLE patients at the time the study was conducted. The frequency of ANA was $77.7 \%$, which is lower than usually reported in SLE patients. However, the prevalence of ANA can change over time. In our patient group, the duration of the disease ranged from 1 to 30 years. Moreover, various immunosuppressive therapeutic schemes were applied in the patients and the majority of them went into remission which also might be the case of ANA disappearance in some of the SLE patients. The analysis of disease histories revealed that at the moment of diagnosis, all of them were positive for ANA.

Serum levels of IL-23 were significantly higher in SLE patients than in the controls $(P=0.0015$ after Bonferroni
TABLE 1: Clinical characteristics of systemic lupus erythematosus patients and healthy controls.

\begin{tabular}{|c|c|c|}
\hline Assessed parameters & $\begin{array}{c}\text { Systemic lupus } \\
\text { erythematosus } \\
\text { patients } \\
n=94 \\
\text { Mean } \pm \text { SD } \\
\text { Median (Q1, Q3) }\end{array}$ & $\begin{array}{c}\text { Healthy } \\
\text { controls } \\
n=27 \\
\text { Mean } \pm \text { SD } \\
\text { Median } \\
\text { (Q1, Q3) }\end{array}$ \\
\hline Age (years) & $44.5 \pm 13.5$ & $43.6 \pm 13.1$ \\
\hline Sex & $\mathrm{F}=82 ; \mathrm{M}=12$ & $\mathrm{~F}=21 ; \mathrm{M}=6$ \\
\hline Disease duration (years) & $7.0(4.0,12.0)$ & - \\
\hline \multicolumn{3}{|l|}{ SLEDAI } \\
\hline Low, $n(\%)$ & $52(55.3 \%)$ & - \\
\hline Medium, $n(\%)$ & $33(35.1 \%)$ & - \\
\hline High, $n(\%)$ & $9(9.6 \%)$ & - \\
\hline APS, $n(\%)$ & $31(33.3 \%)$ & - \\
\hline Lupus nephritis, $n(\%)$ & $24(25.5 \%)$ & - \\
\hline \multicolumn{3}{|l|}{ Cerebrovascular manifestations } \\
\hline TIA, $n(\%)$ & $2(2.1 \%)$ & - \\
\hline Stroke, $n(\%)$ & $10(10.6 \%)$ & - \\
\hline \multicolumn{3}{|l|}{ Cardiovascular manifestations } \\
\hline $\mathrm{CAD}, n(\%)$ & $11(11.7 \%)$ & - \\
\hline MI, $n(\%)$ & $4(4.3 \%)$ & - \\
\hline Raynaud's phenomenon, $n(\%)$ & $25(26.6 \%)$ & - \\
\hline Vasculitis, $n(\%)$ & $14(14.9 \%)$ & - \\
\hline
\end{tabular}

SLEDAI: systemic lupus erythematosus disease activity index; APS: antiphospholipid syndrome; TIA: transient ischemic attacks; CAD: coronary artery disease; MI: myocardial infarction.

correction). The maximum level of IL-23 in the controls was $3.1 \mathrm{pg} / \mathrm{mL}$ and was established as the cut-off value (Table 6 and Figure 1).

The reference range of cIMT was established on the basis of the measurements in the controls. Intima-media thickness were considered moderately increased at values of $0.66-0.86 \mathrm{~mm}$ (51 SLE patients) and highly thickened at $>0.86 \mathrm{~mm}$ (15 SLE patients). Values of $\mathrm{ABI}<1.0$ were found in 22 patients and $<0.9$ in five patients with SLE.

The OR for serum IL-23 $>3.1 \mathrm{pg} / \mathrm{mL}$ in SLE patients with the presence of atherosclerotic plaque in the right common femoral artery was 4.26 (95\% CI 1.16-15.56), $P=0.029$ $(P=0.058$ after Bonferroni correction), and in the arteries of the lower extremities, it was 2.84 (95\% CI 1.02-7.91), $P=0.046(P=0.092$ after Bonferroni correction) (Table 7). The analysis of classical atherosclerotic risk factors revealed that the OR for the serum level of IL-23 $>3.1 \mathrm{pg} / \mathrm{mL}$ in SLE patients with obesity was 3.88 (95\% CI $1.30-11.58$ ), $P=0.015$ ( $P=0.015$ after Bonferroni correction) (Table 7). There was no correlation between IL-23 and cIMT and $\mathrm{ABI}$ values as well as atherosclerotic plaque in the rest of the assessed arteries (data not shown). There was also no correlation between IL-23 and other classical atherosclerotic risk factors (data not shown). The OR for the serum level of IL-23>0.0 pg/mL in SLE patients with lupus nephritis was 3.18 (95\% CI 1.06-9.54), $P=0.039(P=0.039$ after Bonferroni correction) (Table 7). There was no correlation 
TABLE 2: Carotid intima-media thickness, ankle-brachial index, and atherosclerotic plaque in systemic lupus erythematosus patients and healthy controls.

\begin{tabular}{|c|c|c|}
\hline Assessed parameters & $\begin{array}{c}\text { Systemic lupus } \\
\text { erythematosus } \\
\text { patients } \\
n=94 \\
\text { Median (Q1, Q3) }\end{array}$ & $\begin{array}{c}\text { Healthy controls } \\
n=27 \\
\text { Median }(\mathrm{Q} 1, \mathrm{Q} 3)\end{array}$ \\
\hline cIMT (mm) & $0.70(0.65,0.80)$ & $0.60(0.60,0.70)$ \\
\hline $\mathrm{ABI}$ right & $1.08(1.03,1.16)$ & $1.12(1.06,1.24)$ \\
\hline ABI left & $1.08(1.10,1.17)$ & $1.15(1.08,1.21)$ \\
\hline \multicolumn{3}{|l|}{ Plaques, $n(\%)$} \\
\hline cca, $n(\%)$ & $3(3.2 \%)$ & - \\
\hline Bulb, $n(\%)$ & $19(20.2 \%)$ & - \\
\hline ica, $n(\%)$ & $2(2.1 \%)$ & - \\
\hline Carotid arteries, $n(\%)$ & $22(23.4 \%)$ & - \\
\hline iliaca, $n(\%)$ & $8(8.5 \%)$ & - \\
\hline $\mathrm{cfa}, n(\%)$ & $18(19.1 \%)$ & - \\
\hline dfa, $n(\%)$ & $1(1.1 \%)$ & - \\
\hline $\mathrm{sfa}, n(\%)$ & $9(9.6 \%)$ & - \\
\hline popla, $n(\%)$ & $4(4.3 \%)$ & - \\
\hline pta, $n(\%)$ & $1(1.1 \%)$ & - \\
\hline $\begin{array}{l}\text { Lower extremity arteries, } \\
n(\%)\end{array}$ & $27(28.7 \%)$ & - \\
\hline
\end{tabular}

cIMT: carotid intima-media thickness; ABI: ankle-brachial index; cca: common carotid arteries; ica: internal carotid arteries; iliaca: iliac arteries; cfa: common femoral arteries; dfa: deep femoral arteries; sfa: superficial femoral arteries; popla: popliteal arteries; pta: posterior tibial arteries.

between IL-23 and other analyzed clinical complications in SLE patients (data not shown). Moreover, the OR for the serum level of IL-23>0.0 $\mathrm{pg} / \mathrm{mL}$ in SLE patients with the presence of selected autoantibodies such as aPE IgG was 12.67 (95\% CI 1.49-108.06), $P=0.020 \quad(P=0.100$ after Bonferroni correction), aPE IgG or IgM was 4.61 (95\% CI 1.19-17.88), $P=0.027 \quad(P=0.135$ after Bonferroni correction), and aSS-B/La was 11.80 (95\% CI 1.47-94.77), $P=0.020$ ( $P=0.020$ after Bonferroni correction) (Table 7). The OR for the serum level of IL-23>0.0 pg/mL in SLE patients with aCL IgG was 2.26 (95\% CI 0.9-5.71), $P=0.084 \quad(P=0.420$ after Bonferroni correction), and with aPT IgG was 8.35 (95\% CI 0.98-71.09), $P=0.052$ $(P=0.260$ after Bonferroni correction) (Table 7).

In the multivariate stepwise analysis, the $\mathrm{OR}$ for serum levels of IL-23 $>3.1 \mathrm{pg} / \mathrm{mL}$ in SLE patients with atherosclerotic plaque in the right common femoral artery was 4.27 (95\% CI $1.08-16.89), P=0.038$, with obesity was 4.21 (95\% CI 1.40-12.67), $P=0.011$, and with the presence of aSS-B/La was 4.14 (95\% CI 1.14-15.07), $P=$ 0.031 (Table 8).

In the multivariate stepwise analysis, the $\mathrm{OR}$ for the serum level of IL-23>0.0 pg/mL in SLE patients with atherosclerotic plaque in the right common femoral artery was 12.67 (95\% CI $1.41-113.84$ ), $P=0.023$, with obesity was 2.98 (95\% CI $0.84-10.61$ ), $P=0.091$ (result at the border of statistical significance), with lupus nephritis was 3.69 (95\% CI 1.11-12.22), $P=0.033$, with the presence of aSS-B/La
TABLE 3: Laboratory characteristics of systemic lupus erythematosus patients and healthy controls.

\begin{tabular}{|c|c|c|}
\hline Assessed parameters & $\begin{array}{c}\text { Systemic lupus } \\
\text { erythematosus } \\
\text { patients } \\
n=94 \\
\text { Median (Q1, Q3) }\end{array}$ & $\begin{array}{c}\text { Healthy } \\
\text { controls } \\
n=27 \\
\text { Median }(\mathrm{Q} 1, \mathrm{Q} 3)\end{array}$ \\
\hline IL-23 (pg/mL) & $1.20(0.0,3.80)$ & $0.0(0.0,0.0)$ \\
\hline CRP (mg/L) & $2.6(1.2,6.1)$ & $0.0(0.0,1.0)$ \\
\hline $\operatorname{ESR}(\mathrm{mm} / \mathrm{h})$ & $22.0(12.0,45.0)$ & $10.0(2.0,16.0)$ \\
\hline Fibrinogen (mg/dL) & $316.0(271.0,374.5)$ & $283.0(246.5,339.5)$ \\
\hline Homocysteine (mol/L) & $13.9(11.0,18.1)$ & $6.6(5.4,8.1)$ \\
\hline Uric acid $(\mathrm{mg} / \mathrm{dL})$ & $4.6(3.9,5.9)$ & $4.5(3.5,5.3)$ \\
\hline
\end{tabular}

TABLE 4: Classical atherosclerotic risk factors in patients with systemic lupus erythematosus and healthy controls.

\begin{tabular}{|c|c|c|}
\hline Risk factor & $\begin{array}{c}\text { Systemic lupus } \\
\text { erythematosus } \\
\text { patients } \\
n=94 \\
\text { Mean } \pm \text { SD } \\
\text { Median }(\mathrm{Q} 1, \mathrm{Q} 3)\end{array}$ & $\begin{array}{c}\text { Healthy } \\
\text { controls } \\
n=27 \\
\text { Mean } \pm \text { SD } \\
\text { Median } \\
(\mathrm{Q} 1, \mathrm{Q} 3) \\
\end{array}$ \\
\hline Total cholesterol (mg/dL) & $218.5 \pm 59.8$ & $229.3 \pm 41.3$ \\
\hline LDL cholesterol (mg/dL) & $129.1 \pm 47.7$ & $139.3 \pm 34.3$ \\
\hline HDL cholesterol (mg/dL) & $58.9 \pm 24.5$ & $61.3 \pm 9.9$ \\
\hline Triglycerides (mg/dL) & $150.0 \pm 91.2$ & $143.7 \pm 73.3$ \\
\hline Hypertension, $n(\%)$ & $37(39.3 \%)$ & $3(11.1 \%)$ \\
\hline BMI & $25.5 \pm 4.9$ & $24.5 \pm 3.4$ \\
\hline Smoking habits, $n(\%)$ & $31(33.0 \%)$ & $12(44.4 \%)$ \\
\hline Diabetes, $n(\%)$ & $11(11.7 \%)$ & $0(0.0 \%)$ \\
\hline Oral contraceptive use, $n(\%)$ & $4 / 82(4.9 \%)$ & $5 / 21(23.8 \%)$ \\
\hline $\begin{array}{l}\text { Family history of } \\
\text { cardiovascular disease, } n(\%)\end{array}$ & $4(4.3 \%)$ & $4(14.8 \%)$ \\
\hline
\end{tabular}

LDL: low-density lipoprotein; HDL: high-density lipoprotein; BMI: body mass index.

was 15.43 (95\% CI 1.73-137.25), $P=0.014$, and with aPE IgG was 11.06 (95\% CI 1.24-98.65), $P=0.031$ (Table 8).

There was no correlation between IL-23 and other analyzed laboratory and serologic characteristics in SLE patients (data not shown).

\section{Discussion}

The analysis of the role of selected proinflammatory and angiogenic cytokines in SpA patients $[14,15,32,33]$ showed higher levels of IL-23 in ankylosing spondylitis in comparison with the controls and revealed the association with selected clinical characteristics such as psoriatic onycholysis and the use of nonsteroid anti-inflammatory drugs $[14,15]$.

In addition, our initial reports [34] on atherosclerotic risk in SLE patients showed that risk factors for atherosclerosis 
TABLE 5: Serological characteristics of systemic lupus erythematosus patients and healthy controls.

\begin{tabular}{|c|c|c|}
\hline Autoantibody & $\begin{array}{l}\text { Systemic lupus erythematosus patients } \\
\qquad n=94(\%)\end{array}$ & $\begin{array}{c}\text { Healthy controls } \\
n=27(\%)\end{array}$ \\
\hline Antinuclear antibodies IgG & $73(77.7 \%)$ & $2(7.4 \%)$ \\
\hline Anti-double stranded DNA IgG & $37(39.4 \%)$ & $0(0.0 \%)$ \\
\hline Anti-nucleosome IgG & $30(31.9 \%)$ & $0(0.0 \%)$ \\
\hline Anti-Sm IgG & $4(4.3 \%)$ & $0(0.0 \%)$ \\
\hline Anti-SS-A/Ro IgG & $42(44.7 \%)$ & $0(0.0 \%)$ \\
\hline Anti-SS-B/La IgG & $14(14.9 \%)$ & $0(0.0 \%)$ \\
\hline Anti-ribosomal P protein IgG & $6(6.4 \%)$ & $0(0.0 \%)$ \\
\hline Anti-histone IgG & $19(20.2 \%)$ & $0(0.0 \%)$ \\
\hline Anti-U1-RNP IgG & $20(21.3 \%)$ & $0(0.0 \%)$ \\
\hline Anti-cardiolipin IgG & $33(35.1 \%)$ & $0(0.0 \%)$ \\
\hline Anti-cardiolipin IgM & $19(20.2 \%)$ & $1(3.7 \%)$ \\
\hline Anti-cardiolipin IgA & $40(42.6 \%)$ & $1(3.7 \%)$ \\
\hline Anti-beta2-glycoprotein I IgG & $7(7.4 \%)$ & $0(0.0 \%)$ \\
\hline Anti-beta2-glycoprotein I IgM & $23(24.5 \%)$ & $1(3.7 \%)$ \\
\hline Anti-beta2-glycoprotein I IgA & $23(24.5 \%)$ & $0(0.0 \%)$ \\
\hline Anti-oxidized low-density lipoprotein IgG & $45(47.9 \%)$ & $0(0.0 \%)$ \\
\hline Anti-oxidized low-density lipoprotein IgM & $68(72.3 \%)$ & $0(0.0 \%)$ \\
\hline Anti-prothrombin IgG & $10(10.6 \%)$ & $0(0.0 \%)$ \\
\hline Anti-prothrombin IgM & $12(12.8 \%)$ & $0(0.0 \%)$ \\
\hline Anti-prothrombin IgA & $11(11.7 \%)$ & $0(0.0 \%)$ \\
\hline Anti-phosphatidylserine IgG & $7(7.4 \%)$ & $0(0.0 \%)$ \\
\hline Anti-phosphatidylserine IgM & $6(6.4 \%)$ & $1(3.7 \%)$ \\
\hline Anti-phosphatidylethanolamine IgG & $12(12.8 \%)$ & $0(0.0 \%)$ \\
\hline Anti-phosphatidylethanolamine IgM & $6(6 \%)$ & $0(0.0 \%)$ \\
\hline Lupus anticoagulant & $15(16.0 \%)$ & $0(0.0 \%)$ \\
\hline Anti-neutrophil cytoplasmic antibodies IgG & $39(41.5 \%)$ & $2(7.4 \%)$ \\
\hline Anti-proteinase 3 IgG & $0(0.0 \%)$ & $0(0.0 \%)$ \\
\hline Anti-myeloperoxidase IgG & $9(9.6 \%)$ & $0(0.0 \%)$ \\
\hline Anti-lactoferrin IgG & $12(12.8 \%)$ & $0(0.0 \%)$ \\
\hline Anti-elastase IgG & $9(9.6 \%)$ & $0(0.0 \%)$ \\
\hline Anti-BPI IgG & $1(1.1 \%)$ & $0(0.0 \%)$ \\
\hline Anti-endothelial cell antibody IgG & $42(44.7 \%)$ & $3(11.1 \%)$ \\
\hline
\end{tabular}

BPI: bactericidal/permeability-increasing protein.

TABLE 6: Serum levels of IL-23 in patients with systemic lupus erythematosus and controls.

\begin{tabular}{|c|c|c|c|c|c|}
\hline Serum level of IL-23 (pg/mL) & Systemic lupus erythematosus patients, $n(\%)$ & Controls, $n(\%)$ & OR $(95 \% \mathrm{CI})$ & $P$ & $P^{\#}$ \\
\hline 0.0 & $39(41.4)$ & $22(81.8)$ & 1.00 & & \\
\hline $0.01-3.10$ & $30(31.9)$ & $5(18.5)$ & $3.38(1.07-12.64)$ & 0.0223 & 0.0669 \\
\hline$>3.10$ & $25(26.6)$ & $0(0.0)$ & $14.1(3.55-60.48)$ & 0.0005 & 0.0015 \\
\hline
\end{tabular}

IL-23: interleukin-23; OR: odds ratio; 95\% CI: 95\% confidence interval; ${ }^{*} P$ : after Bonferroni correction. OR and $P$ adjusted for sex and age.

in SLE differ from those observed in the general population. Chronic inflammation, the presence of selected autoantibodies (e.g., aPLs and AECA), and applied treatment (especially high cumulative dosage of glucocorticosteroids) are particularly important in atherosclerosis development. However, there is still the need to search for other pathomechanisms underlying this process.
In this study, we evaluated the relationship between serum levels of IL-23 and clinical and laboratory characteristics of SLE with the special focus on atherosclerosis and atherosclerotic risk factors. We found a significant correlation between high serum levels of IL-23 and atherosclerotic changes in the arteries of the lower extremity arteries. This finding stays in accordance with the reports on the IL-23 


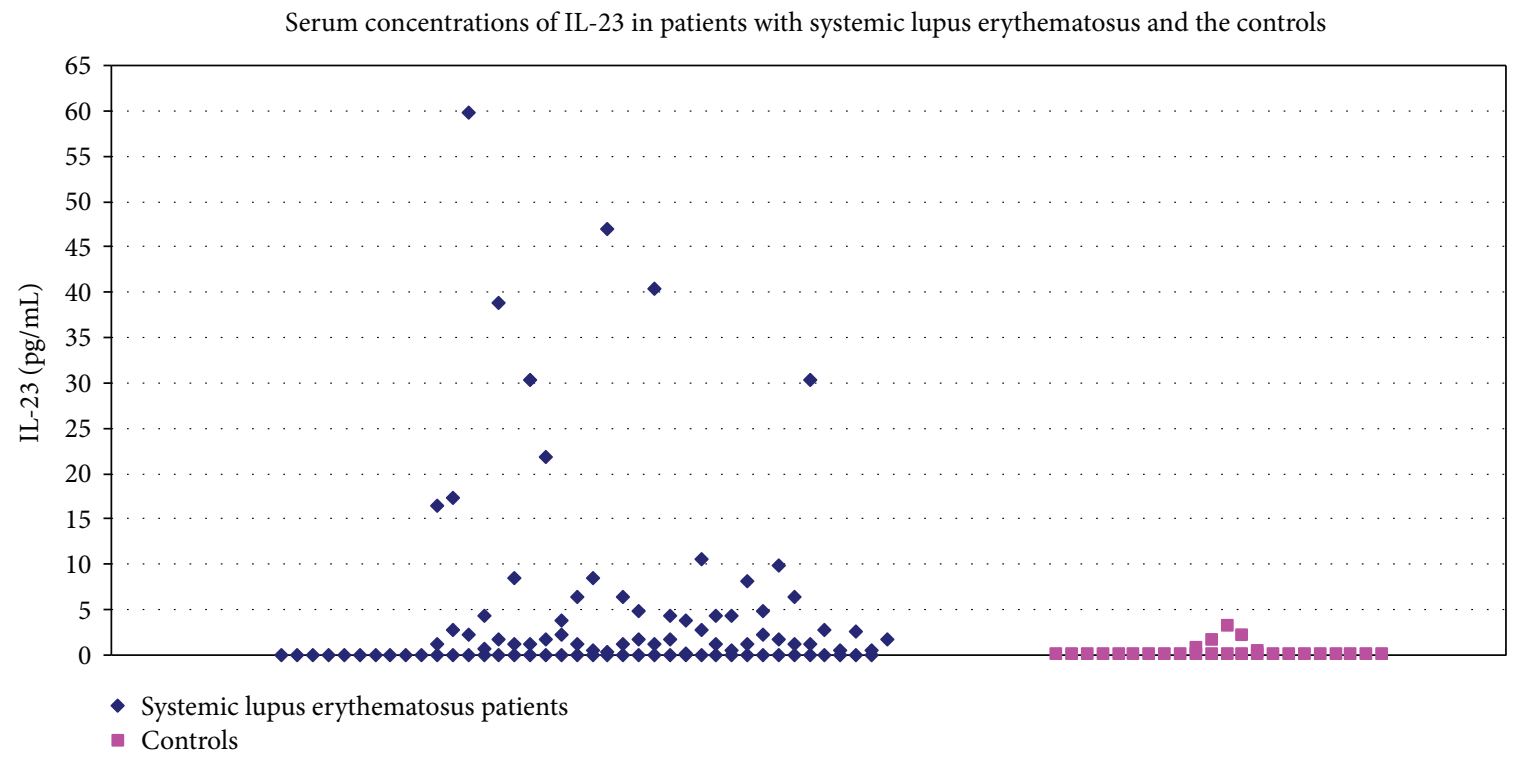

Figure 1: Serum concentrations of IL-23 in patients with systemic lupus erythematosus and the controls.

TABLE 7: A logistic regression model of the OR of the increased serum level of IL-23 in systemic lupus erythematosus patients.

\begin{tabular}{|c|c|c|c|c|c|c|}
\hline \multirow{2}{*}{ Covariates } & \multicolumn{3}{|c|}{ Serum IL-23 $>0.0 \mathrm{pg} / \mathrm{mL}$} & \multicolumn{3}{|c|}{ Serum IL-23 $>3.1 \mathrm{pg} / \mathrm{mL}$} \\
\hline & OR $(95 \% \mathrm{CI})$ & $P$ & $P^{\#}$ & OR $(95 \% \mathrm{CI})$ & $P$ & $P^{\#}$ \\
\hline Atherosclerotic plaque in cfa & $10.12(1.20-85.14)$ & 0.033 & 0.066 & $4.26(1.16-15.66)$ & 0.029 & 0.058 \\
\hline Atherosclerotic plaque in lower extremity arteries in general & $1.26(0.48-3.32)$ & 0.640 & 1.000 & $2.84(1.02-7.91)$ & 0.046 & 0.092 \\
\hline Obesity & $3.83(1.19-12.29)$ & 0.024 & 0.024 & $3.88(1.30-11.58)$ & 0.015 & 0.015 \\
\hline Lupus nephritis & $3.18(1.06-9.54)$ & 0.039 & 0.039 & $1.61(0.52-4.97)$ & 0.406 & 0.406 \\
\hline Anti-phosphatidylethanolamine IgG & $12.67(1.49-108.06)$ & 0.020 & 0.100 & $0.71(0.13-3.82)$ & 0.687 & 1.000 \\
\hline Anti-phosphatidylethanolamine IgG or IgM & $4.61(1.19-17.88)$ & 0.027 & 0.135 & $1.01(0.28-3.62)$ & 0.983 & 1.000 \\
\hline Anti-SS-B/La IgG & $11.80(1.47-94.77)$ & 0.020 & 0.020 & $5.21(1.53-17.71)$ & 0.008 & 0.008 \\
\hline Anti-cardiolipin IgG & $2.26(0.90-5.71)$ & 0.084 & 0.420 & $1.66(0.61-4.49)$ & 0.322 & 1.000 \\
\hline Anti-prothrombin IgG & $8.35(0.98-71.09)$ & 0.052 & 0.260 & $0.64(0.12-3.40)$ & 0.598 & 1.000 \\
\hline
\end{tabular}

IL-23: interleukin-23; cfa: common femoral arteries; OR: odds ratio; 95\% CI: 95\% confidence interval; ${ }^{*} P$ : after Bonferroni correction. OR and $P$ adjusted for sex and age.

TABLE 8: The multivariate stepwise analysis for serum levels of IL-23>3.1 pg/mL and IL-23>0.0 pg/mL.

\begin{tabular}{|c|c|c|c|c|}
\hline \multirow{2}{*}{ Covariates } & \multicolumn{2}{|c|}{ Serum IL-23 > 3.1 pg/mL } & \multicolumn{2}{|c|}{ Serum IL-23 $>0.0 \mathrm{pg} / \mathrm{mL}$} \\
\hline & OR $(95 \% \mathrm{CI})$ & $P$ & OR $(95 \% \mathrm{CI})$ & $P$ \\
\hline Atherosclerotic plaque in cfa & $4.27(1.08-16.89)$ & 0.038 & $12.67(1.41-113.84)$ & 0.023 \\
\hline Obesity & $4.21(1.40-12.67)$ & 0.011 & $2.98(0.84-10.61)$ & 0.091 \\
\hline Anti-SS-B/La IgG & $4.14(1.14-15.07)$ & 0.031 & $15.43(1.73-137.25)$ & 0.014 \\
\hline Lupus nephritis & NS & NS & $3.69(1.11-12.22)$ & 0.033 \\
\hline Anti-phosphatidylethanolamine IgG & NS & NS & $11.06(1.24-98.65)$ & 0.031 \\
\hline
\end{tabular}

Cfa: common femoral artery; NS: nonsignificant. OR and $P$ adjusted for sex and age.

serum levels in patients affected by peripheral arterial disease (PAD) [22]. The authors analyzed 29 patients with advanced PAD: 28 had stage IV and one had stage III disease. All patients were qualified for lower extremity bypass (LEB) surgery using either autogenous vein or synthetic polytetrafluoroethylene (PTFE) graft material. Blood for measurement of IL-23 levels was taken at three points: $24 \mathrm{~h}$ before,
$24 \mathrm{~h}$ after, and 5 days after the surgical intervention. IL-23 levels were higher at all three time points compared to the control group. Interestingly, 22 patients undergoing LEB with the autogenous vein had IL-23 levels greater than the seven patients who had synthetic PTFE graft material at all three time points. In our study, we did not find a significant correlation between serum levels of IL-23 and ABI values. 
This might be the result of the low number of patients with clinical manifestation of PAD. However, the number of SLE patients with $\mathrm{ABI}<1.0$ was 22 , but significantly decreased values of this index $<0.9$ were found only in three patients, and among them, two confirmed intermittent claudication symptoms and only one underwent surgical intervention. In any case, the significant association between IL-23 levels and the presence of atherosclerotic plaque in lower extremity arteries, especially common femoral arteries, supports the hypothesis that IL-23 might be involved in PAD development in the course of SLE. Furthermore, there are data in the literature that address the possible role of the IL-23-IL17 axis in carotid atherosclerosis [21] and in CAD [35].

Abbas et al. [21] showed that patients with carotid atherosclerosis had markedly raised plasma levels of IL-23 compared with healthy controls. The highest levels were present in patients with the most recent symptoms within the last two months. Moreover, IL-23 concentrations at follow-up after several years (mean 3.5) were associated with increased mortality mainly caused by cerebrovascular and cardiovascular events.

Khojasteh-Fard et al. [35] found a significant decrease of IL-23 gene expression in unstimulated peripheral blood lymphocytes of patients with CAD compared to those without CAD reinforcing the potential role of IL-23 in the complex mechanisms associated with the development of atherosclerosis. Data from patients who underwent diagnostic catheterization showed imbalances between Th17 and regulatory T cells and significantly higher levels of serum IL-23 along with IL-17 and IL-6 in those with acute MI and unstable angina compared with concentrations in patients with stable angina and chest pain syndrome [36]. Our study did not reveal a significant correlation of IL-23 serum levels and CAD, MI, stroke, and TIA as well as cIMT in SLE patients. To our knowledge, there are no available data in the literature concerning this issue. Some studies showed increased levels of IL-6 in SLE patients and the association with the burden of atherosclerosis [37]. Similarly, SLE patients with atherosclerosis showed increased levels of IL-6 and IL-17 compared with SLE alone and the controls [38]. The exact role of IL-23 and the IL-23-IL-17 axis in atherosclerosis and the development of complications in SLE patients still remains the issue that should be the matter of further studies.

In this study, we also analyzed the correlation between serum levels of IL-23 and classical atherosclerotic risk factors in SLE patients. We only found a significant association between IL-23 levels and obesity. Data from the study in 26 obese women and 20 lean volunteers showed significant increases of IL-23 levels as well as IL-17, leptin, and macrophage migration inhibitory factor (MIF) in obese subjects compared with the controls. Interestingly, there was no relationship between IL-12 and interferon $\gamma$ and obesity. Moreover, the authors did not find the correlation between IL-23 and IL-17 and central obesity markers like BMI and waist circumference in obese women that suggests that the accumulation of IL-23-IL-17-producing cells in adipose tissue might not increase in proportion with the buildup of abdominal fat. It is also possible that blood mononuclear cells or some other cell type, rather than leukocytes infiltrating the adipose tissue, could be the main source of IL-17 and IL-23 in obese subjects. The role of the IL-23-IL-17 axis seems to be increased in obese women independently of proinflammatory mediators MIF and leptin [23]. These observations together with the data on the possible role of the IL-23-IL17 proinflammatory cytokine axis in atherosclerosis development may indicate the important link between obesity and cardiovascular complications in SLE patients, and further research should be conducted to investigate this problem.

The association between SLE activity and clinical characteristics of the disease and IL-23 levels was also studied. We found significantly higher levels of IL-23 in SLE patients than in the controls, and there was also an important correlation of IL-23 serum concentrations and lupus nephritis, which is in accordance with other data. The relationship between the elevated serum levels of IL-23 and renal involvement in SLE patients was confirmed by several studies [39-41]. High serum IL-23 mRNA levels were also found in patients with SLE and renal disease [20]. Similarly, higher levels of serum IL-23 were found in SLE patients compared with the controls $[17,36,42]$. We did not confirm the relationship between SLE activity and other clinical characteristics of the disease. In the literature, contrary data are available on the association of IL-23 and SLE activity. Some reports showed a correlation between high levels of IL-23 and activity of the disease [18] as well as a relationship between IL-23 levels and selected manifestations like serositis and cutaneous involvement among patients with active disease [19]. Some reports presented discrepant results and did not show any association of serum IL-23 and SLE activity $[12,17,36]$. In our study, only nine patients had high activity of the disease according to the SLEDAI scale. The majority had low (52) or medium (33) activity, which may partly explain the lack of a relationship between IL-23 and activity of SLE.

Another interesting finding in our study was the significant correlation between serum IL-23 and selected autoantibodies including aPLs and aSS-B/La. Little data are available on the association between IL-23 and the serologic profile in SLE patients. Some studies did not find any correlation of IL23 with autoantibodies typical for lupus like anti-double stranded DNA or anti-Sm $[18,19]$. In fact, only the basic profile of autoantibodies was analyzed in these studies. One paper focused on IL-17 single-nucleotide polymorphisms and increased activity of the IL-23-IL-17 cytokine axis including serum measurements of IL-17 and IL-23, and tissue growth factor $\beta$ in PAPS confirmed higher levels of IL-23 than that in controls. A significant correlation between IL-17 and IL-23 was reported. IL-17 was more frequently found in patients with deep vein thrombosis and thrombocytopenia. No significant associations between the analyzed cytokines and aPLs or clinical manifestations of PAPS were found [13]. In our previous observations in SLE patients with atherosclerotic changes, we found significant relationships between aPLs, secondary antiphospholipid syndrome, and atherosclerotic lesions [34, 43]. In this regard, the current finding is important to explain the possible mechanism of their involvement in atherosclerosis pathogenesis in SLE 
patients. Further studies are warranted to fully analyze the role of the IL-17-IL-23 cytokine axis in this phenomenon.

\section{Conclusion}

In conclusion, there are data, including ours, that support the involvement of IL-23 in SLE pathogenesis. Higher levels of IL-23 in patients compared with the controls and a significant correlation between IL-23 levels and selected clinical characteristics such as lupus nephritis were documented. The limitation of our study was that we analyzed only IL23 serum levels, without the broad spectrum of proinflammatory cytokines. A relatively low number of patients presented cardiovascular and cerebrovascular manifestations. The majority of patients showed low activity of the disease. Even though we were able to confirm the interesting relationship between atherosclerotic lesions in the arteries of the lower extremities, obesity as well as selected aPLs and IL-23 levels in lupus patients also suggests the possible contribution of this cytokine in vascular involvement in the course of SLE, revealing new insights into atherothrombotic risk assessment in SLE patients. Further studies are necessary to fully elucidate this issue.

\section{Conflicts of Interest}

The authors declare that they have no competing interests.

\section{Authors' Contributions}

Katarzyna Fischer participated in the design and coordination of the study, performed the statistical analysis and all immunological tests, and drafted the manuscript. Hanna Przepiera-Będzak and Marek Brzosko participated in the design and coordination of the study and helped to draft the manuscript. Iwona Brzosko helped to collect clinical data. Anna Walecka and Marcin Sawicki performed the imaging investigations.

\section{References}

[1] C. Bombardier, D. D. Gladman, M. B. Urowitz et al., "Derivation of the SLEDAI. A disease activity index for lupus patients," Arthritis \& Rheumatism, vol. 35, no. 6, pp. 630640, 1992.

[2] M. Molokhia and P. McKeigue, "Systemic lupus erythematosus: genes versus environment in high risk populations," Lupus, vol. 15, no. 11, pp. 827-832, 2006.

[3] L. A. Criswell, "The genetic contribution to systemic lupus erythematosus," Bulletin of the NYU Hospital for Joint Diseases, vol. 66, no. 3, pp. 176-183, 2008.

[4] Y. Shoenfeld, D. Alarcon-Segovia, D. Buskila et al., "Frontiers of SLE: review of the 5th International Congress of Systemic Lupus Erythematosus, Cancun, Mexico, April 20-25, 1998," Seminars in Arthritis \& Rheumatism, vol. 29, no. 2, pp. 112130, 1999.

[5] L. E. Muñoz, K. Lauber, M. Schiller, A. A. Manfredi, and M. Herrmann, "The role of defective clearance of apoptotic cells in systemic autoimmunity," Nature Reviews Rheumatology, vol. 6, no. 5, pp. 280-289, 2010.
[6] D. Yan Hin Yap and K. Neng Lai, "Cytokines and their roles in the pathogenesis of systemic lupus erythematosus: from basics to recent advances," Journal of Biomedicine and Biotechnology, vol. 2010, Article ID 365083, 10 pages, 2010.

[7] B. Oppmann, R. Lesley, B. Blom et al., "Novel p19 protein engages IL-12p40 to form a cytokine, IL-23, with biological activities similar as well as distinct from IL-12," Immunity, vol. 13, no. 5, pp. 715-725, 2000.

[8] C. Parham, M. Chirica, J. Timans et al., "A receptor for the heterodimeric cytokine IL-23 is composed of IL-12R $\beta 1$ and a novel cytokine receptor subunit, IL-23R," The Journal of Immunology, vol. 168, no. 11, pp. 5699-5708, 2002.

[9] E. Duvallet, L. Semerano, E. Assier, G. Falgarone, and M. C. Boissier, "Interleukin-23: a key cytokine in inflammatory diseases," Annals of Medicine, vol. 43, no. 7, pp. 503-511, 2011.

[10] Y. Iwakura and H. Ishigame, "The IL-23/IL-17 axis in inflammation," The Journal of Clinical Investigation, vol. 116, no. 5, pp. 1218-1222, 2006.

[11] K. Komura, M. Fujimoto, M. Hasegawa et al., "Increased serum interleukin 23 in patients with systemic sclerosis," The Journal of Rheumatology, vol. 35, no. 1, pp. 120-125, 2008.

[12] R. M. Talaat, S. F. Mohamed, I. H. Bassyouni, and A. A. Raouf, "Th1/Th2/Th17/Treg cytokine imbalance in systemic lupus erythematosus (SLE) patients: correlation with disease activity," Cytokine, vol. 72, no. 2, pp. 146-153, 2015.

[13] D. Popovic-Kuzmanovic, I. Novakovic, L. Stojanovich et al., "Increased activity of interleukin-23/interleukin-17 cytokine axis in primary antiphospholipid syndrome," Immunobiology, vol. 218, no. 2, pp. 186-191, 2013.

[14] H. Przepiera-Będzak, K. Fischer, and M. Brzosko, "Serum IL-6 and IL-23 levels and their correlation with angiogenic cytokines and disease activity in ankylosing spondylitis, psoriatic arthritis, and SAPHO syndrome," Mediators of Inflammation, vol. 2015, Article ID 785705, 7 pages, 2015.

[15] H. Przepiera-Będzak, K. Fischer, and M. Brzosko, "Extra-articular symptoms in constellation with selected serum cytokines and disease activity in spondyloarthritis," Mediators of Inflammation, vol. 2016, Article ID 7617954, 7 pages, 2016.

[16] M. Mohammadi, M. M. Hayatbakhsh, M. J. Zahedi, M. R. Jalalpour, and A. Pakgohar, "Serum interleukin-23 levels in patients with ulcerative colitis," Iranian Journal of Immunology, vol. 8, no. 3, pp. 183-188, 2011.

[17] C. K. Wong, L. C. W. Lit, L. S. Tam, E. K. M. Li, P. T. Y. Wong, and C. W. K. Lam, "Hyperproduction of IL-23 and IL-17 in patients with systemic lupus erythematosus: implications for Th17-mediated inflammation in auto-immunity," Clinical Immunology, vol. 127, no. 3, pp. 385-393, 2008.

[18] D. Salah Hegab, M. Mahmoud Gamei, W. Mohamed Saudi, D. Ezzat Abou Ammo, M. Mohamed El Bedewy, and N. Fathy Elhabian, "Role of interleukin-23 in the immunopathogenesis of systemic lupus erythematosus," Egyptian Journal of Dermatology and Venerology, vol. 34, no. 2, pp. 120-125, 2014.

[19] M. Yin Mok, H. Jing Wu, Y. Lo, and C. Sing Lau, “The relation of interleukin 17 (IL-17) and IL-23 to Th1/Th2 cytokines and disease activity in systemic lupus erythematosus," The Journal of Rheumatology, vol. 37, no. 10, pp. 2046-2052, 2010.

[20] J. Du, Z. Li, J. Shi, and L. Bi, "Associations between serum interleukin-23 levels and clinical characteristics in patients with systemic lupus erythematosus," Journal of International Medical Research, vol. 42, no. 5, pp. 1123-1130, 2014. 
[21] A. Abbas, I. Gregersen, S. Holm et al., "Interleukin 23 levels are increased in carotid atherosclerosis. Possible role for interleukin 23/interleukin 17 axis," Stroke, vol. 46, no. 3, pp. 793799, 2015.

[22] A. David, S. Saitta, G. De Caridi et al., "Interleukin-23 serum levels in patients affected by peripheral arterial disease," Clinical Biochemistry, vol. 45, no. 3, pp. 275-278, 2012.

[23] M. Sumarac-Dumanovic, D. Stevanovic, A. Ljubic et al., "Increased activity of interleukin-23/interleukin-17 proinflammatory axis in obese women," International Journal of Obesity, vol. 33, no. 1, pp. 151-156, 2009.

[24] M. C. Hochberg, "Updating the American College of Rheumatology revised criteria for the classification of systemic lupus erythematosus," Arthritis and Rheumatism, vol. 40, no. 9, p. $1725,1997$.

[25] S. Miyakis, M. D. Lockshin, T. Atsumi et al., "International consensus statement on an update of the classification criteria for definite antiphospholipid syndrome (APS)," Journal of Thrombosis and Haemostasis, vol. 4, no. 2, pp. 295-306, 2006.

[26] G. Howard, A. R. Sharrett, G. Heiss et al., "Carotid artery intimal-medial thickness distribution in general populations as evaluated by B-mode ultrasound. ARIC Investigators," Stroke, vol. 24, no. 9, pp. 1297-1304, 1993.

[27] S. Ebrahim, O. Papacosta, P. Whincup et al., "Carotid plaque, intima media thickness, cardiovascular risk factors, and prevalent cardiovascular disease in men and women," Stroke, vol. 30, no. 4, pp. 841-850, 1999.

[28] S. Homma, N. Hirose, H. Ishida, T. Ishii, and G. Araki, "Carotid plaque and intima-media thickness assessed by Bmode ultrasonography in subjects ranging from young adults to centenarians," Stroke, vol. 32, no. 4, pp. 830-835, 2001.

[29] K. Fischer, "Risk factors of thickened intima-media and atherosclerotic plaque development in carotid arteries in patients with systemic lupus erythematosus," Annales Academiae Medicae Stetinensis, vol. 54, no. 2, pp. 22-32, 2008.

[30] P. G. Vlachoyiannopoulos, P. G. Kanellopoulos, J. P. A. Ioannidis, M. G. Tektonidou, I. Mastorakou, and H. M. Moutsopoulos, "Atherosclerosis in premenopausal women with antiphospholipid syndrome and systemic lupus erythematosus: a controlled study," Rheumatology, vol. 42, no. 5, pp. 645-651, 2003.

[31] D. Sacks, C. W. Bakal, P. T. Beatty et al., "Position statement on the use of the anklebrachial index in the evaluation of patients with peripheral vascular disease," Journal of Vascular and Interventional Radiology, vol. 13, p. 1353, 2002.

[32] H. Przepiera-Będzak, K. Fischer, and M. Brzosko, "Serum levels of angiogenic cytokines in psoriatic arthritis and SAPHO syndrome," Polish Archives of Internal Medicine, vol. 123, no. 6, pp. 297-302, 2013.

[33] H. Przepiera-Będzak, K. Fischer, and M. Brzosko, "Serum VEGF, EGF, basic FGF, and acidic FGF levels and their association with disease activity and extra-articular symptoms in ankylosing spondylitis," Polish Archives of Internal Medicine, vol. 126, no. 4, pp. 290-292, 2016.

[34] K. Fischer and M. Brzosko, "Diagnosis of early atherosclerotic lesions, and selected atherosclerotic risk factors, in patients with systemic lupus erythematosus," Polish Archives of Internal Medicine, vol. 119, no. 11, pp. 736-741, 2009.

[35] M. Khojasteh-Fard, M. Abolhalaj, P. Amiri et al., "IL-23 gene expression in PBMCs of patients with coronary artery disease," Disease Markers, vol. 33, no. 6, pp. 289-293, 2012.
[36] X. Cheng, X. Yu, Y. J. Ding et al., “The Th17/Treg imbalance in patients with acute coronary syndrome," Clinical Immunology, vol. 127, no. 1, pp. 89-97, 2008.

[37] Y. Asanuma, C. P. Chung, A. Oeser et al., "Increased concentration of proatherogenic inflammatory cytokines in systemic lupus erythematosus: relationship to cardiovascular risk factors," The Journal of Rheumatology, vol. 33, no. 3, pp. 539-545, 2006.

[38] M. Zhu, H. Mo, X. Li, and L. Zhang, "Th17/Treg imbalance induced by increased incidence of atherosclerosis in patients with systemic lupus erythematosus (SLE)," Clinical Rheumatology, vol. 32, no. 7, pp. 1045-1052, 2013.

[39] D.-Y. Chen, Y.-M. Chen, M.-C. Wen, T.-Y. Hsieh, W.-T. Hung, and J.-L. Lan, "The potential role of Th17 cells and Th17related cytokines in the pathogenesis of lupus nephritis," Lupus, vol. 21, no. 13, pp. 1385-1396, 2012.

[40] A. Zickert, P. Amoudruz, Y. Sundström, J. Rönnelid, V. Malmström, and I. Gunnarsson, "IL-17 and IL-23 in lupus nephritis - association to histopathology and response to treatment," BMC Immunology, vol. 16, no. 7, p. 10, 2015.

[41] L. P. Xia, B. F. Li, H. Shen, and J. Lu, "Interleukin-27 and interleukin-23 in patients with systemic lupus erythematosus: possible role in lupus nephritis," Scandinavian Journal of Rheumatology, vol. 44, no. 3, pp. 200-205, 2015.

[42] F. Qiu, L. Song, N. Yang, and X. Li, "Glucocorticoid downregulates expression of IL-12 family cytokines in systemic lupus erythematosus patients," Lupus, vol. 22, no. 10, pp. 10111016, 2013.

[43] A. Walecka, M. Sawicki, M. Brzosko, L. Ostanek, K. Fischer, and J. Kordowski, "Value of high resistance index - HRI calculated from Doppler spectrum of popliteal arteries in patients with systemic lupus erythematosus (SLE)," Medical Science Monitor, vol. 10, Supplement 3, pp. 58-62, 2004. 


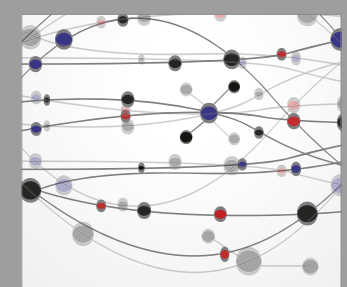

The Scientific World Journal
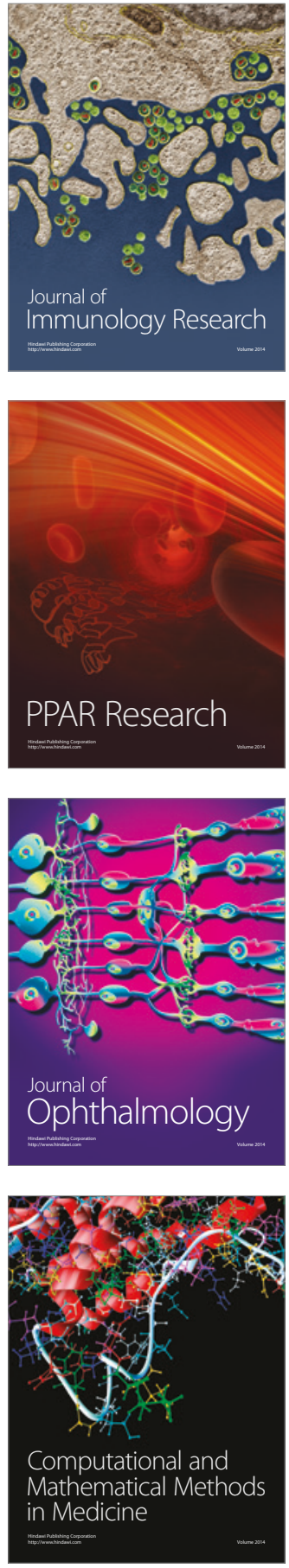

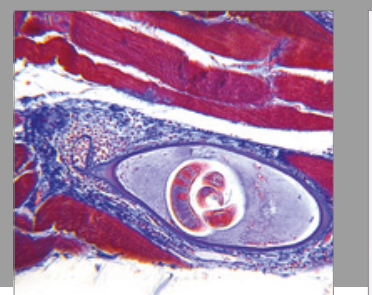

Gastroenterology Research and Practice
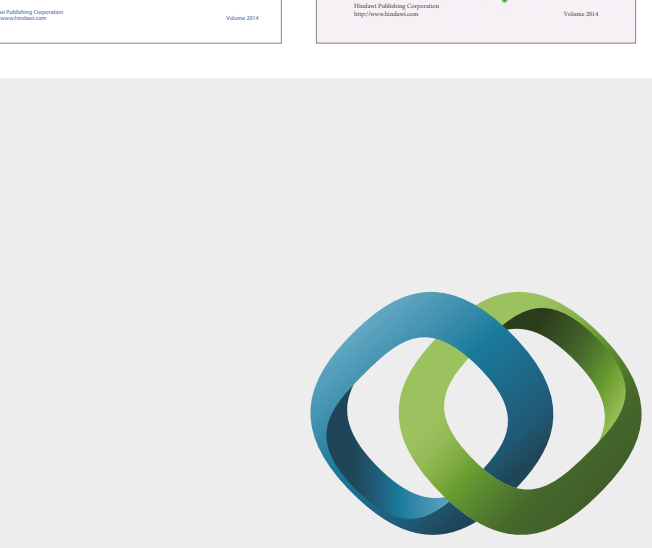

\section{Hindawi}

Submit your manuscripts at

https://www.hindawi.com
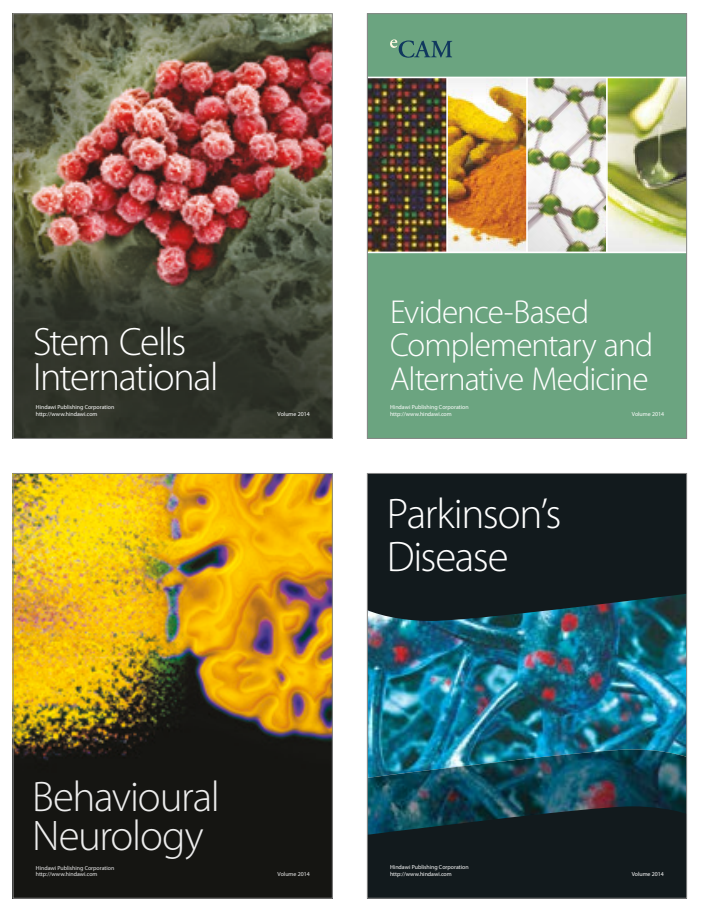
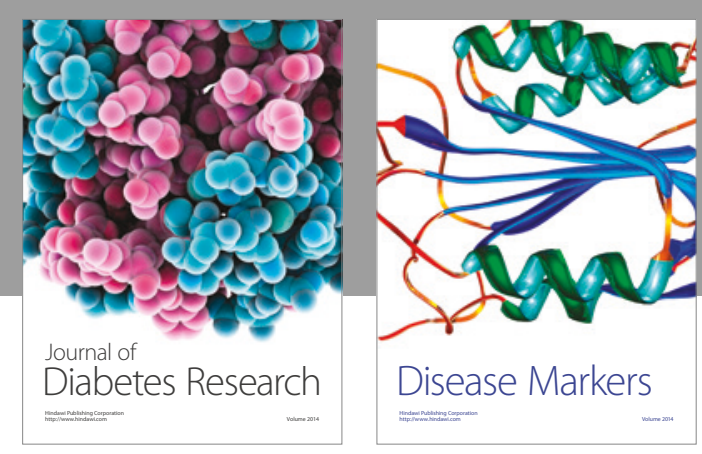

Disease Markers
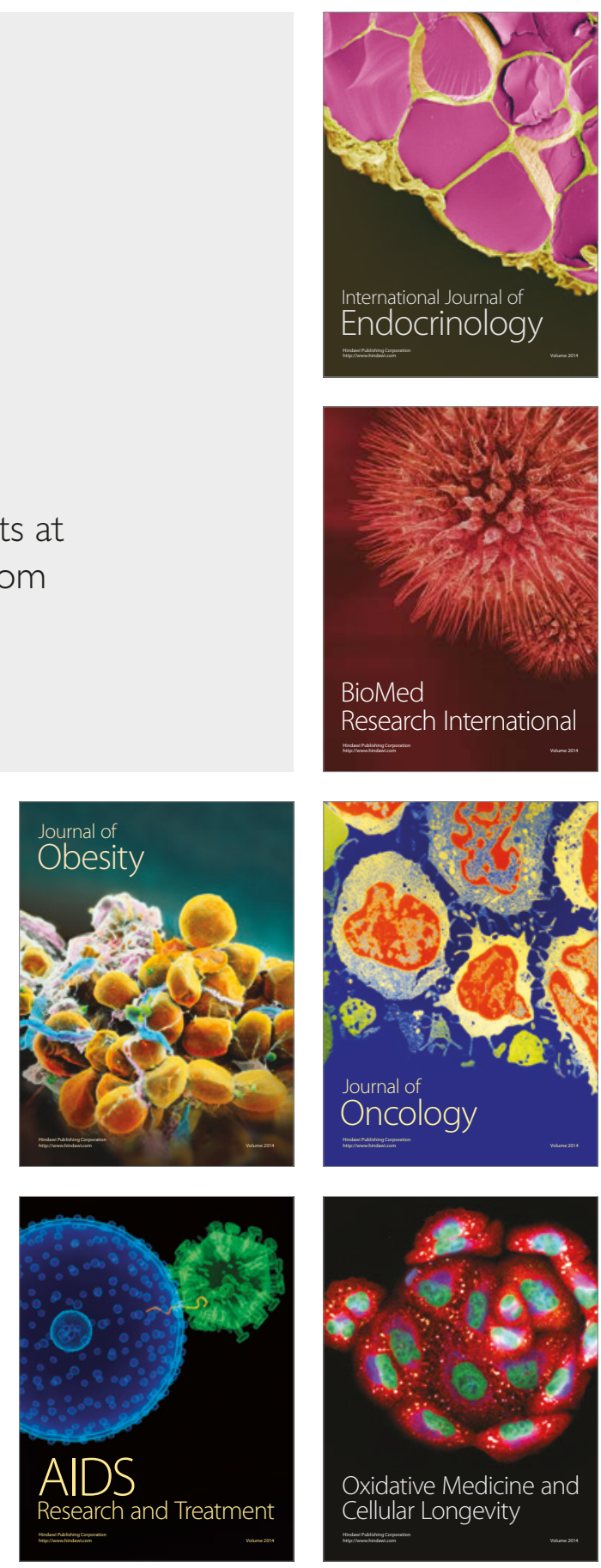